\title{
Reversible hydrogenation of deuterium-intercalated quasi-free-standing graphene on $\mathrm{SiC}(0001)$
}

\author{
F. C. Bocquet, ${ }^{1, *}$ R. Bisson, ${ }^{2, \dagger}$ J.-M. Themlin, ${ }^{1}$ J.-M. Layet, ${ }^{2}$ and T. Angot ${ }^{2}$ \\ ${ }^{1}$ Aix-Marseille Univ, IM2NP, 13397 Marseille, France and CNRS, UMR 7334, 13397 Marseille-Toulon, France \\ ${ }^{2}$ Aix-Marseille Univ, PIIM, CNRS, UMR 7345, 13397 Marseille, France
}

(Received 7 February 2012; revised manuscript received 18 April 2012; published 8 May 2012)

\begin{abstract}
Hydrogenation of deuterium-intercalated quasi-free-standing monolayer graphene on $\mathrm{SiC}(0001)$ is obtained and studied with low-energy electron diffraction and high-resolution electron energy loss spectroscopy. While the carbon honeycomb structure remains intact, it is shown that a significant band gap opens in the hydrogenated material. Vibrational spectroscopy evidences for hydrogen chemisorption on the quasi-free-standing graphene is provided and its thermal stability is studied.
\end{abstract}

DOI: 10.1103/PhysRevB.85.201401

PACS number(s): 81.05.ue, 68.43.Pq, 79.20.Uv

Graphene, a zero-gap semiconductor, presents outstanding physical properties and raises hope for a new electronic era. The use of a semiconducting substrate to support graphene is of importance both for its applications ${ }^{1}$ and for the understanding of its fundamental properties. ${ }^{2}$ To that purpose, silicon carbide (SiC) appeared as an ideal substrate for the growth of largescale and high quality graphene. ${ }^{3}$ Graphene can be grown on $\mathrm{SiC}$ by high-temperature annealing in inert atmosphere or ultrahigh vacuum (UHV). ${ }^{3,4}$ The first step is the creation of the $(6 \sqrt{3} \times 6 \sqrt{3}) R 30^{\circ}$ reconstructed interface layer, the so-called buffer layer, a carbon honeycomb anchored to the $\mathrm{SiC}$ substrate by covalent bonds. By increasing the annealing temperature, one or several graphene layers are grown atop the buffer layer. However, monolayer graphene obtained by this way is coupled to the buffer layer and is $n$ doped, its Dirac cone being offset by $\sim 0.4 \mathrm{eV}$ from the Fermi energy level $\left(E_{F}\right){ }^{5,6}$ Recently, Riedl et al. ${ }^{7}$ devised a method to prepare from $\mathrm{SiC}$ a single layer graphene with the Dirac cone located at $E_{F}$ and most of the outstanding properties of exfoliated graphene flakes are approached. ${ }^{8}$ This method consists of intercalating hydrogen atoms $(\mathrm{H})$ at the buffer layer interface by a mild heating of the substrate in hydrogen atmosphere ${ }^{7}$ or in UHV under $\mathrm{H}$ atom flux. ${ }^{9}$ First principles calculations ${ }^{10}$ and photoelectron ${ }^{7}$ and infrared ${ }^{8}$ spectroscopies have shown that the buffer layer bonds to $\mathrm{SiC}$ are broken and capped with $\mathrm{H}$ and the buffer layer becomes a quasi-free-standing monolayer graphene, a single layer of graphite efficiently decoupled from the substrate. ${ }^{6,8}$

A simple method to open a band gap or to induce a metal-to-insulator transition on supported graphene is highly desirable to design graphene-based devices. To date, this has been demonstrated by hydrogenation of the graphene layer on metallic substrates, such as $\mathrm{Ir}^{11}$ and $\mathrm{Au} / \mathrm{Ni}^{12}$ In this Rapid Communication, we provide vibrational spectroscopy evidences of the successful hydrogenation of quasi-freestanding monolayer graphene on a semiconducting substrate, $\mathrm{SiC}(0001)$, accompanied by a loss of graphene metallicity.

Experiments were conducted in a UHV preparation chamber (base pressure $<2 \times 10^{-10}$ mbar) equipped with a hydrogen atom source of the hot capillary type (Omicron EFM$\mathrm{H})$ and connected to a high-resolution electron energy loss spectroscopy (HREELS) analysis chamber (VSI Delta 0.5, base pressure $\left.<1 \times 10^{-10} \mathrm{mbar}\right)$. Samples of $\sim 0.7 \mathrm{~cm}^{2}$ surface were prepared from semi-insulating $6 \mathrm{H}-\mathrm{SiC}(0001)$ wafers (TankeBlue, carrier concentration $\sim 10^{15} \mathrm{~cm}^{-3}$ ). We prepared the buffer layer by first obtaining the Si-rich $\mathrm{SiC}-(3 \times 3)$ surface, subsequently annealed to $1450 \mathrm{~K} .{ }^{13}$ We assessed the quality of the buffer layer (avoiding the formation of coupled graphene atop) by verifying a low-energy electron diffraction (LEED) pattern on the entire surface (see the Supplemental Material $^{14}$ and Refs. 6,9,13,15, and 16 therein). Deuterium intercalation, transforming the buffer layer in quasi-freestanding monolayer graphene (denoted as SiC-D/QFMLG), was performed with a $\mathrm{D}$ atom exposure of $\sim 5 \times 10^{17} \mathrm{~cm}^{-2}$ at a surface temperature of $950 \mathrm{~K}$ and confirmed by LEED and scanning tunneling microscopy (STM). ${ }^{6,14}$ Finally, hydrogenation up to saturation of quasi-free-standing monolayer graphene was performed at room temperature with a $\mathrm{H}$ atom exposure $>3 \times 10^{15} \mathrm{~cm}^{-2}$. The latter sample is denoted as $\mathrm{SiC}-\mathrm{D} / \mathrm{QFMLG}-\mathrm{H}$ to stress the different isotopes used, first, to prepare quasi-free-standing monolayer graphene (the D-intercalation step), and second, to hydrogenate it. As it will be shown in the following, the obtaining of deuteriumintercalated and hydrogenated quasi-free-standing monolayer graphene, as well as samples made at intermediate preparation steps, are clearly distinguishable with HREELS measurements performed at room temperature.

Figure 1 shows characteristic HREEL spectra taken in specular geometry $\left(65^{\circ}\right.$ angle of incidence with respect to the surface normal) with a $5 \mathrm{eV}$ electron primary beam energy for the buffer layer, SiC-D/QFMLG and SiC-D/QFMLG-H samples. In addition to the elastic peak, the three spectra present the well known Fuchs-Kliewer (FK) SiC optical phonon at an electron energy loss of about $115 \mathrm{meV}$ as well as FK phonon overtones/multiple losses at $\sim 230$ and $\sim 345 \mathrm{meV}$ (denoted as $2 \mathrm{FK}$ and $3 \mathrm{FK}$, respectively). The obvious difference between those spectra are the different widths of the energy loss features and the corresponding decreasing background which extends to energy losses up to $500 \mathrm{meV}$ and higher. We characterize these differences by measuring the elastic peak's full width at half maximum (FWHM) as shown in Fig. 1. Dielectric theory is known to properly describe HREELS in specular geometry. ${ }^{17}$ In particular, FWHM and the shape of the HREELS elastic peak are strongly influenced by the electronic properties of the layers interacting with the electron primary beam. Therefore, the change of FWHM we observe in Fig. 1 between the buffer layer, SiC-D/QFMLG, and $\mathrm{SiC}-\mathrm{D} / \mathrm{QFMLG}-\mathrm{H}$ is a measure of the modification of electronic structure between the three samples. 


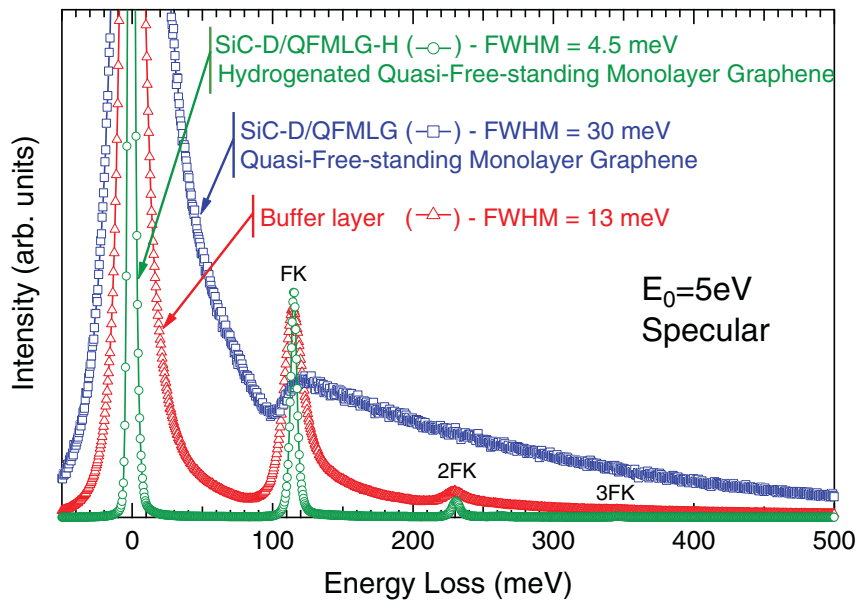

FIG. 1. (Color online) HREEL spectra of the buffer layer (red triangle), SiC-D/QFMLG (blue square) and SiC-D/QFMLG-H (green circle) samples.

The buffer layer sample has a semiconducting surface with a band gap of at least $1.6 \mathrm{eV}$ containing faint surface states located between 0.1 and $0.5 \mathrm{eV}$ and around $0.9 \mathrm{eV}$ below $E_{F} \cdot{ }^{6,18}$ Guisinger et al.${ }^{19}$ have shown that these surface states disappear upon hydrogenation of the buffer layer. We found that the HREELS FWHM of the buffer layer drops from 13 to $4.5 \mathrm{meV}$ when the sample is hydrogenated (not shown), the latter value being typical of intrinsic semiconducting surfaces with the settings of our spectrometer. Therefore we conclude that the major source of FWHM broadening in the buffer layer is related to its surface states.

Upon D intercalation, the buffer layer transforms in SiCD/QFMLG, ${ }^{6,14}$ which translates in HREELS as an increase of the elastic peak FWHM from 13 to $30 \mathrm{meV}$. In this case, the broadening of the elastic peak is obviously related to the vanishing band gap of graphene, since FK phonon losses are efficiently screened by the quasi-free-standing graphene monolayer (Fig. 1). We note that the FK loss of SiC-D/QFMLG does not present a two-peak structure, in contrast to what is observed for monolayer graphene grown atop the buffer layer. ${ }^{20}$ This absence of FK phonon-plasmon coupling is further evidence of the efficient decoupling from the substrate obtained with deuterium intercalation. Furthermore, the elastic peak asymmetry in the SiC-D/QFMLG spectra is markedly more important than in the case of the buffer layer. We tentatively attribute this asymmetry to the Drude damping ${ }^{21}$ of a plasmon feature located at the loss energy of $\sim 5.8 \mathrm{eV}$, as observed in specular geometry for SiC-D/QFMLG but absent in the buffer layer sample (primary beam energy $26 \mathrm{eV}$, not shown). In summary, the large and asymmetric HREELS elastic peak observed for the SiC-D/QFMLG is rationalized by the presence of the delocalized $\pi$ and $\pi^{\star}$ bands up to $E_{F}$ of the graphene layer.

Hydrogenation of suspended graphene as well as quasifree-standing graphene supported on Ir or $\mathrm{Au} / \mathrm{Ni}$ is known to open a wide band gap around $E_{F}$ while keeping the integrity of the carbon honeycomb structure. ${ }^{11,12,22}$ Figure 1 shows that upon hydrogenation of SiC-D/QFMLG, the obtained SiC-D/QFMLG-H sample presents a dramatic decrease of its peaks FWHM, from 30 to $4.5 \mathrm{meV}$. The latter FWHM is similar to the one measured for the hydrogenated semiconducting buffer layer sample, i.e., in the absence of surface states. Furthermore, 2FK and 3FK phonon losses reappeared and the $\sim 5.8 \mathrm{eV}$ plasmon feature disappeared. Finally, the LEED pattern of the SiC-D/QFMLG-H remains very similar to the one of SiC-D/QFMLG. ${ }^{14}$ Taken together, these observations show that, upon hydrogen atom exposure, the graphene lattice structure and its positioning on the $\mathrm{SiC}$ substrate is unchanged but its metallic character is lost. We measured the evolution of the elastic peak width as a function of the $\mathrm{H}$ dose (from $2 \times 10^{13} \mathrm{~cm}^{-2}$ up to saturation, i.e., $>3 \times 10^{15} \mathrm{~cm}^{-2}$ ). A monotonic decrease of the FWHM is observed, indicating that the electronic density of states (DOS) near $E_{F}$ is smoothly decreasing with hydrogen coverage, consistent with the observation of a band-gap opening of hydrogenated graphene on Ir. ${ }^{11}$ However, a precise characterization of the physical mechanism behind the loss of metallicity is beyond the scope of this study.

In addition to the features of Fig. 1 discussed above, the SiC-D/QFMLG-H spectrum presents several supplementary peaks not visible in the present scale. These peaks and others can be better seen when HREELS is performed in a out-ofspecular geometry, where the dipolar lobe induced background is strongly reduced. ${ }^{23}$ In Fig. 2, we display spectra of two hydrogenated graphene on $\mathrm{SiC}$ obtained with inverted isotopic preparation, i.e., SiC-D/QFMLG-H and SiC-H/QFMLG-D samples. The isotopic inversion permits to unambiguously attribute energy loss peaks to a chemical bonding by looking at the quantitative oscillator frequency shift when one of the two oscillator partners is changed (in the present case Si-D to $\mathrm{Si}-\mathrm{H}$ or C-H to C-D inversion).

Apart from FK SiC phonon losses and the LO phonon of graphene at $193 \mathrm{meV},{ }^{24,25}$ constant in frequency, several peaks are found to shift upon isotopic inversion. We highlight those shift in Fig. 2 by arrows, the ones pointing down (respectively up) highlighting the C-H to C-D (respectively $\mathrm{Si}-\mathrm{H}$ to $\mathrm{Si}-\mathrm{D}$ ) substitution. A summary of spectroscopic signatures and their assignments are presented in Table I. On the SiC-H/QFMLG-D

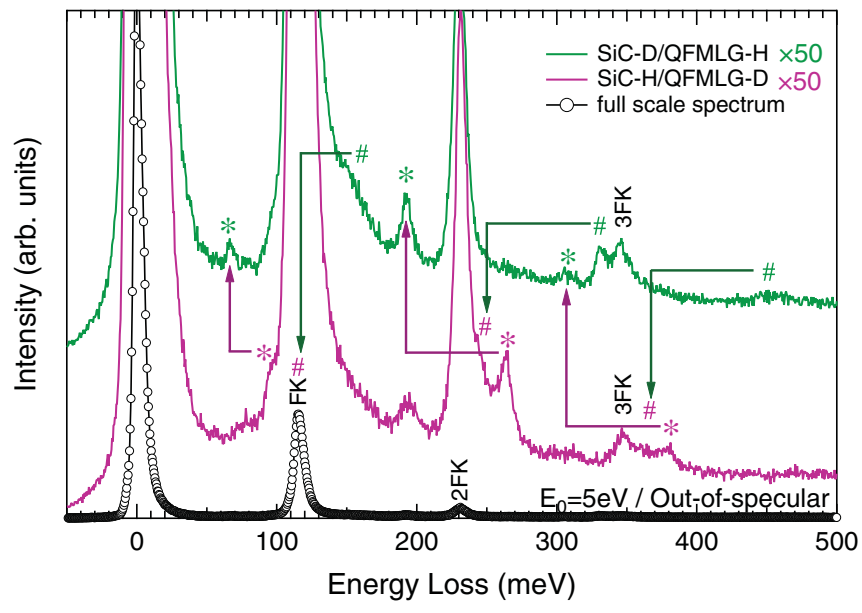

FIG. 2. (Color online) HREEL spectra of SiC-D/QFMLG-H (top trace) and SiC-H/QFMLG-D (middle trace) samples. Si and C related vibrations are marked with star $(*)$ and sharp (\#) symbols, respectively. Arrows indicate peaks shifts due to $\mathrm{H} \rightarrow \mathrm{D}$ isotope substitution. 
TABLE I. Summary of HREELS vibrational peak assignment based on isotopic inversion. $\delta$ and $v$ stand for bending and stretching vibrational modes, respectively. Mode frequencies are given in $\mathrm{meV}$.

\begin{tabular}{lccccc}
\hline \hline Sample & Mode & Si-D & Si-H & C-D & C-H \\
\hline SiC-D/QFMLG-H & $\delta$ & 67 & & & 153 \\
& $v$ & 193 & & & 331 \\
& $v+$ FK & 306 & & & 449 \\
SiC-H/QFMLG-D & $\delta$ & & 95 & FK & \\
& $v$ & & 263 & 243 & \\
& $v+$ FK & & 378 & 358 & \\
\hline \hline
\end{tabular}

(respectively SiC-D/QFMLG-H) sample, the peak located at $263 \mathrm{meV}$ (respectively $193 \mathrm{meV}$, on top of the LO phonon) is attributed to the signature of intercalated hydrogen bound to the $\mathrm{Si}$ atoms of the $\mathrm{SiC}$ substrate. Although slightly shifted to higher frequency as compared to the $\mathrm{Si}-\mathrm{H}$ (respectively $\mathrm{Si}$-D) stretch on silicon surfaces, ${ }^{26}$ the energy loss we attribute to a $\mathrm{Si}-\mathrm{H}$ stretch is consistent with the infrared adsorption measurement of Speck et al. ${ }^{8}$ and the Si-D to Si-H frequency shift agrees quantitatively to the change of oscillator mass. Also shifted to higher frequency as compared to silicon surfaces, the peak at $95 \mathrm{meV}$ (respectively $67 \mathrm{meV}$ ) is assigned to the $\mathrm{Si}-\mathrm{H}$ (respectively $\mathrm{Si}-\mathrm{D}$ ) bending mode. The peak at $378 \mathrm{meV}$ (respectively $306 \mathrm{meV}$ ) results from the combination between the $\mathrm{Si}-\mathrm{H}$ (respectively $\mathrm{Si}-\mathrm{D}$ ) stretch and the FK SiC phonon. On the SiC-D/QFMLG-H (respectively SiC-H/QFMLG-D) sample, peaks observed at 331 and $153 \mathrm{meV}$ (respectively $243 \mathrm{meV}$ and in the FK phonon) coincides with stretch and bend vibrations of hydrogen (respectively deuterium) dimers on graphite. ${ }^{27,28}$ Similarly to the case of Si-H, the peak at $449 \mathrm{meV}$ (respectively $358 \mathrm{meV}$ ) is assigned to the combination between the $\mathrm{C}-\mathrm{H}$ (respectively $\mathrm{C}-\mathrm{D})$ stretch and the FK $\mathrm{SiC}$ phonon.

The analysis of out-of-specular HREEL spectra provides several evidences. For the sake of clarity, we will discuss the SiC-D/QFMLG-H sample in the following. First, the observation of Si-D oscillators confirms the nature of the intercalation used to obtain quasi-free-standing graphene on $\mathrm{SiC}$, i.e., the intercalated deuterium is covalently bound to the silicon atoms of the $\mathrm{SiC}$ substrate, consistent with the recent infrared spectroscopy measurement on SiC-H/QFMLG by Speck et al. ${ }^{8}$ It is therefore reasonable to assume that prior to intercalation, the buffer layer binding is made between $\mathrm{Si}$ atoms of the $\mathrm{SiC}$ and atoms of the carbon honeycomb layer, as most of recent calculations have assumed. ${ }^{29,30}$ Second, the observation of $\mathrm{C}-\mathrm{H}$ oscillators proves the successful hydrogenation of quasi-free-standing graphene on $\mathrm{SiC}$ and is consistent with the present observation of a drastically reduced DOS near $E_{F} .{ }^{31-33}$ The measured C-H and C-D frequencies (Table I) are in good agreement with the adsorption clustering in dimers calculated by several groups on single side hydrogenated isolated graphene ${ }^{28,34}$ and is consistent with STM observations on graphite ${ }^{35}$ and $n$-doped graphene grown atop of the buffer layer. ${ }^{19,36}$ We note that Kim et al. have measured $\mathrm{C}-\mathrm{H}$ and C-D frequencies for hydrogenated graphene supported on $\operatorname{Ir}(111),{ }^{37}$ lower than the calculated ones on single side hydrogenated isolated graphene. In light of our results, we suggest that the metal substrate has a significant influence on

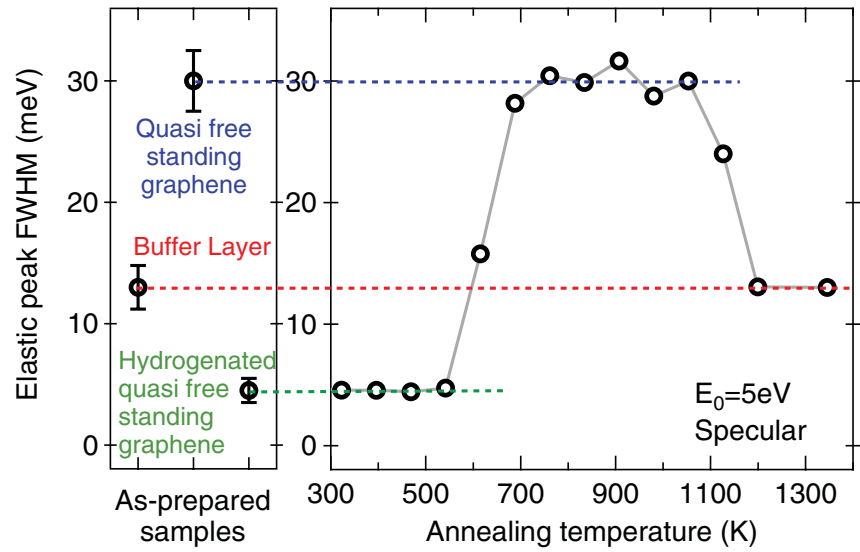

FIG. 3. (Color online) Evolution of the HREELS elastic peak FWHM of SiC-D/QFMLG-H upon annealing. The annealing temperature uncertainty is estimated to be $\pm 5 \%$. Error bars represent the $\pm \sigma$ variation of FWHM measured across the entire surface of several samples.

hydrogen adsorption on $\operatorname{Ir}(111)$, in contrast to SiC-D/QFMLG$\mathrm{H}$ samples which present a hydrogenated quasi-free-standing graphene efficiently decoupled from the SiC-D substrate. Third, we recall that SiC-D/QFMLG-H samples are obtained with two steps of hydrogen isotope exposure performed at different sample temperatures. The first step is the deuterium intercalation below the graphene performed at $950 \mathrm{~K}$ and the second step is the hydrogen adsorption on graphene performed at $300 \mathrm{~K}$. Close inspection of Fig. 2 shows that the second isotope exposure at room temperature does not change the nature of the intercalation oscillator, i.e., extended exposure of SiC-D/QFMLG to hydrogen atoms at $300 \mathrm{~K}$ produces $\mathrm{SiC}-\mathrm{D} / \mathrm{QFMLG}-\mathrm{H}$ and not $\mathrm{SiC}-\mathrm{H} / \mathrm{QFMLG}-\mathrm{H}$. Isotope substitution by abstraction and subsequent adsorption is known to be efficient on silicon surfaces ${ }^{38}$ and we did observe isotope substitution of the intercalated deuterium when performing the intercalation step a second time with changing isotope, i.e., SiC-D/QFMLG becomes SiC-H/QFMLG when exposed to hydrogen atoms at $950 \mathrm{~K}$. Therefore, we demonstrate that $\mathrm{D}$-intercalated quasi-free-standing graphene on $\mathrm{SiC}$ is impenetrable to hydrogen atoms at $300 \mathrm{~K}$.

This raises the question of why the deuterium intercalation step needs to be performed at an elevated sample temperature. In order to investigate this matter, we have tested, first, the stability of hydrogenated quasi-free-standing graphene on $\mathrm{SiC}$ upon thermal treatment. SiC-D/QFMLG-H samples were gradually annealed in UHV and their evolution was followed with LEED and the elastic peak FWHM of HREELS recorded in specular geometry. In Fig. 3, we observe the transformation of SiC-D/QFMLG-H into SiC-D/QFMLG at a sample temperature of $600 \mathrm{~K}$ that we relate to hydrogen desorption from the graphene. Then, when the sample is heated above $1100 \mathrm{~K}$, intercalated deuterium desorbs and the graphene layer is anchored back to the $\mathrm{SiC}$, recreating the buffer layer, consistent with the observation of Riedl et al. ${ }^{7}$ The recovered buffer layer can be used again to prepare (hydrogenated) quasifree-standing graphene. Second, we exposed the buffer layer sample to hydrogen atoms at room temperature and performed LEED and HREELS measurements as a function of annealing 
temperature. Adsorbed hydrogen atoms on the buffer layer are found to be remarkably stable upon sample annealing, desorbing from the buffer layer only above $\sim 750 \mathrm{~K}$. Therefore, we conclude that preparation of quasi-free-standing graphene on $\mathrm{SiC}$ by hydrogen intercalation is possible in a small sample temperature window because of the competition between hydrogen chemisorption on the buffer layer ( $<750 \mathrm{~K})$, hydrogen intercalation below the buffer layer $(750-1100 \mathrm{~K})$, and hydrogen desorption from the intercalation site $(>1100 \mathrm{~K})$. The exact intercalation mechanism (hydrogen diffusion through the anchored graphene lattice, at defect or at boundary of the anchored graphene layer) remains an open question.
In summary, we have obtained hydrogenated deuteriumintercalated quasi-free-standing graphene on $\mathrm{SiC}$ and shown its stability in vacuum up to $550 \mathrm{~K}$. Hydrogenated quasifree-standing graphene conserves its honeycomb structure and possesses a significant band gap, allowing HREEL spectroscopy to probe the nature of chemical bonds below the graphene sheet. Intercalation and hydrogenation-induced band-gap opening are reversible processes, a promising feature for the processing of technological devices.

F.C.B. and J.-M.T. gratefully acknowledge support from the project ANR 2010-Blanc 1017 "ChimigraphN."
*Present address: Peter Grünberg Institut (PGI-3), Forschungszentrum Jülich, 52425 Jülich, Germany.

${ }^{\dagger}$ Corresponding author: regis.bisson@ polytechnique.edu

${ }^{1}$ Y. M. Lin, C. Dimitrakopoulos, K. A. Jenkins, D. B. Farmer, H. Y. Chiu, A. Grill, and P. Avouris, Science 327, 662 (2010).

${ }^{2}$ G. M. Rutter, J. N. Crain, N. P. Guisinger, T. Li, P. N. First, and J. A. Stroscio, Science 317, 219 (2007).

${ }^{3}$ K. V. Emtsev, A. Bostwick, K. Horn, J. Jobst, G. L. Kellogg, L. Ley, J. L. McChesney, T. Ohta, S. A. Reshanov, J. Roehrl, E. Rotenberg, A. K. Schmid, D. Waldmann, H. B. Weber, and T. Seyller, Nat. Mater. 8, 203 (2009).

${ }^{4}$ J. Hass, W. A. de Heer, and E. H. Conrad, J. Phys.: Condens. Matter 20, 323202 (2008).

${ }^{5}$ T. Ohta, A. Bostwick, J. L. McChesney, T. Seyller, K. Horn, and E. Rotenberg, Phys. Rev. Lett. 98, 206802 (2007).

${ }^{6}$ C. Riedl, C. Coletti, and U. Starke, J. Phys. D: Appl. Phys. 43, 374009 (2010).

${ }^{7}$ C. Riedl, C. Coletti, T. Iwasaki, A. A. Zakharov, and U. Starke, Phys. Rev. Lett. 103, 246804 (2009).

${ }^{8}$ F. Speck, J. Jobst, F. Fromm, M. Ostler, D. Waldmann, M. Hundhausen, H. B. Weber, and T. Seyller, Appl. Phys. Lett. 99, 122106 (2011).

${ }^{9}$ S. Watcharinyanon, C. Virojanadara, J. R. Osiecki, A. A. Zakharov, R. Yakimova, R. I. G. Uhrberg, and L. I. Johansson, Surf. Sci. 605, 1662 (2011).

${ }^{10}$ A. Mattausch and O. Pankratov, Phys. Rev. Lett. 99, 076802 (2007).

${ }^{11}$ R. Balog, B. Jorgensen, L. Nilsson, M. Andersen, E. Rienks,

M. Bianchi, M. Fanetti, E. Laegsgaard, A. Baraldi, S. Lizzit,

Z. Sljivancanin, F. Besenbacher, B. Hammer, T. G. Pedersen,

P. Hofmann, and L. Hornekaer, Nat. Mater. 9, 315 (2010).

${ }^{12}$ D. Haberer, D. V. Vyalikh, S. Taioli, B. Dora, M. Farjam, J. Fink, D. Marchenko, T. Pichler, K. Ziegler, S. Simonucci, M. S. Dresselhaus, M. Knupfer, B. Buechner, and A. Grueneis, Nano Lett. 10, 3360 (2010).

${ }^{13}$ I. Forbeaux, J.-M. Themlin, and J.-M. Debever, Phys. Rev. B 58, 16396 (1998).

${ }^{14}$ See Supplemental Material at http://link.aps.org/supplemental/ 10.1103/PhysRevB.85.201401 for details on samples preparation and supporting LEED and STM measurements.

${ }^{15}$ H. Hibino, H. Kageshima, F. Maeda, M. Nagase, Y. Kobayashi, and H. Yamaguchi, Phys. Rev. B 77, 075413 (2008).

${ }^{16}$ S. Forti, K. V. Emtsev, C. Coletti, A. A. Zakharov, C. Riedl, and U. Starke, Phys. Rev. B 84, 125449 (2011).

${ }^{17}$ P. Lambin, L. Henrard, P. Thiry, C. Silien, and J. Vigneron, J. Electron Spectrosc. Relat. Phenom. 129, 281 (2003).
${ }^{18}$ K. V. Emtsev, F. Speck, T. Seyller, L. Ley, and J. D. Riley, Phys. Rev. B 77, 155303 (2008).

${ }^{19}$ N. P. Guisinger, G. M. Rutter, J. N. Crain, P. N. First, and J. A. Stroscio, Nano Lett. 9, 1462 (2009).

${ }^{20}$ R. J. Koch, T. Seyller, and J. A. Schaefer, Phys. Rev. B 82, 201413 (2010).

${ }^{21}$ T. Angot and G. Gewinner, J. Electron Spectrosc. Relat. Phenom. 104, 173 (1999).

${ }^{22}$ D. C. Elias, R. R. Nair, T. M. G. Mohiuddin, S. V. Morozov, P. Blake, M. P. Halsall, A. C. Ferrari, D. W. Boukhvalov, M. I. Katsnelson, A. K. Geim, and K. S. Novoselov, Science 323, 610 (2009).

${ }^{23}$ H. Ibach and D. L. Mills, Electron Energy Loss Spectroscopy and Surface Vibrations (Academic, New York, 1982).

${ }^{24}$ T. Aizawa, R. Souda, S. Otani, Y. Ishizawa, and C. Oshima, Phys. Rev. B 42, 11469 (1990).

${ }^{25}$ W. H. Soe, K. H. Rieder, A. M. Shikin, V. Mozhaiskii, A. Varykhalov, and O. Rader, Phys. Rev. B 70, 115421 (2004).

${ }^{26}$ M. Eremtchenko, F. Tautz, R. Öttking, and J. Schaefer, Surf. Sci. 600, 3446 (2006).

${ }^{27}$ T. Zecho, A. Güttler, X. Sha, B. Jackson, and J. Küppers, J. Chem. Phys. 117, 8486 (2002).

${ }^{28}$ A. Allouche, Y. Ferro, T. Angot, C. Thomas, and J.-M. Layet, J. Chem. Phys. 123, 124701 (2005).

${ }^{29}$ B. Lee, S. Han, and Y.-S. Kim, Phys. Rev. B 81, 075432 (2010).

${ }^{30}$ O. Pankratov, S. Hensel, and M. Bockstedte, Phys. Rev. B 82, 121416 (2010)

${ }^{31}$ S. Casolo, O. M. Lovvik, R. Martinazzo, and G. F. Tantardini, J. Chem. Phys. 130, 054704 (2009).

${ }^{32}$ J. Zhou, Q. Wang, Q. Sun, X. S. Chen, Y. Kawazoe, and P. Jena, Nano Lett. 9, 3867 (2009).

${ }^{33}$ B. S. Pujari, S. Gusarov, M. Brett, and A. Kovalenko, Phys. Rev. B 84, 041402 (2011).

${ }^{34}$ S. Sakong and P. Kratzer, J. Chem. Phys. 133, 054505 (2010).

${ }^{35}$ L. Hornekær, Z. Šljivančanin, W. Xu, R. Otero, E. Rauls, I. Stensgaard, E. Lægsgaard, B. Hammer, and F. Besenbacher, Phys. Rev. Lett. 96, 156104 (2006).

${ }^{36}$ R. Balog, B. Jørgensen, J. Wells, E. Lægsgaard, P. Hofmann, F. Besenbacher, and L. Hornekær, J. Am. Chem. Soc. 131, 8744 (2009).

${ }^{37}$ H. Kim, T. Balgar, and E. Hasselbrink, Chem. Phys. Lett. 508, 1 (2011).

${ }^{38}$ A. Dinger, C. Lutterloh, and J. Küppers, Chem. Phys. Lett. 311, 202 (1999). 